\title{
Evaluating the effectiveness of two methods to improve students' problem solving performance after studying an online tutorial
}

\author{
Zhongzhou Chen, ${ }^{1}$ Kyle M. Whitcomb, ${ }^{2}$ Matthew W. Guthrie, ${ }^{1}$ and Chandralekha Singh ${ }^{2}$ \\ ${ }^{1}$ Department of Physics, University of Central Florida, Orlando, FL, 32816 \\ ${ }^{2}$ Department of Physics and Astronomy, University of Pittsburgh, Pittsburgh, PA, 15260
}

\begin{abstract}
An earlier study using sequences of online learning modules found that a significant fraction of undergraduate students were unable to solve similar new problems after learning from an online problem solving tutorial. The current study examines the effectiveness of two methods to improve students' subsequent problem solving performance. First, an "on-ramp" module designed to help students develop proficiency in relevant basic skills was added prior to the tutorial. We found that students' performance on subsequent modules improved significantly over the previous year, and in one of the two sequences we found evidence to support that the improvement was due to the addition of the on-ramp module rather than other irrelevant factors. Second, a new "transfer" module was added after the tutorial and before the final quiz module in which half of the students were given a comparecontrast task and the other half were asked to answer tutorial-style scaffolding questions. On the subsequent quiz module, we did not find significant performance differences between the two conditions, nor did students' performance significantly improve over the previous year. The study demonstrated that mastery-style online homework can serve as an efficient and flexible method for evaluating the effectiveness of new instructional designs.
\end{abstract}

2019 PERC Proceedings edited by Cao, Wolf, and Bennett; Peer-reviewed, doi.org/10.1119/perc.2019.pr.Chen Published by the American Association of Physics Teachers under a Creative Commons Attribution 4.0 license. Further distribution must maintain attribution to the article's authors, cover page, and DOI. 


\section{INTRODUCTION}

Studies in both general human problem solving and problem solving in physics have long shown that it is difficult for novices to transfer the understanding and skills learned in one problem context to a different, new context [1-3]. In physics, it is well known that novices tend to focus more on superficial differences between problems and pay less attention to deep structural similarities between problems [4]. In an earlier study involving a sequence of three online learning modules, we observed that while most introductory-level college physics students learned to solve a specific problem after engaging with an online problem solving tutorial in the first module, student performance when solving similar problems on two subsequent modules was either unchanged or only slightly improved [5]. In the current study, we tested two strategies for improving student performance on subsequent similar problems following an online tutorial.

First, research in both learning science [6-8] and physics education $[9,10]$ have shown that explicitly comparing and contrasting multiple examples can be more effective for understanding the common deep structure of the problems compared to studying isolated examples in sequence. The theory predicts that explicitly asking students to compare a new problem to a previously solved similar problem results in better performance on subsequent transfer tasks than only asking students to study features of the new problem.

Second, students' performance in our previous study could have been negatively impacted by a lack of sufficient mastery of one or more basic skills [11,12] such as identifying the direction of angular momentum using the right hand rule. Students who are not fluent in those skills may have to devote too many cognitive resources while executing those procedures, leaving insufficient cognitive capacity to process the deeper structure of the problem. Alternatively, students could have implemented a correct problem-solving procedure with a small mistake such as a sign error in one of the steps leading to an incorrect answer. In either case, performance on subsequent similar problems could improve if they had the opportunity to practice and strengthen their basic skills.

Therefore, we will investigate the following two research questions:

RQ1. Does answering several compare-contrast style questions lead to better performance on a subsequent transfer task compared to completing several guided-tutorial style questions?

RQ2. Does the addition of an "on-ramp" module designed to develop basic procedural skills improve students' performance on subsequent problem solving tasks?

Both research questions will be answered by analyzing data collected from students' interactions with a sequence of online learning modules, which are assigned as regular homework for students to complete over a period of two weeks. Since students' performance on online homework problems could be impacted by other extraneous factors (e.g., copying answers from various sources $[13,14]$ ), we will also take

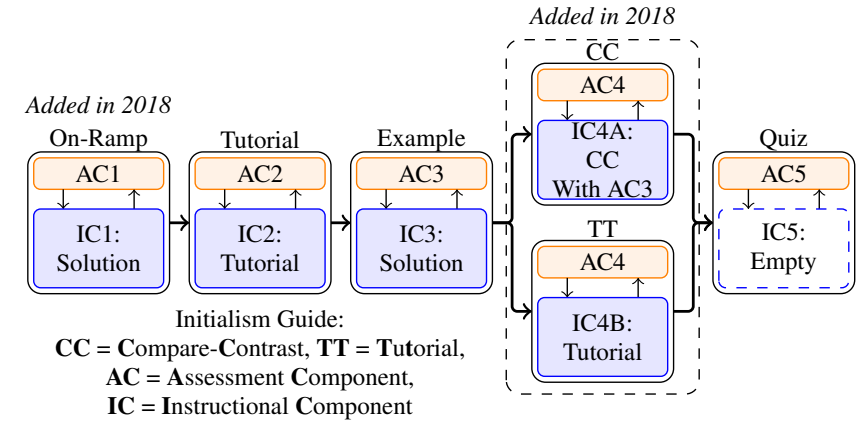

FIG. 1. The sequence of Online Learning Modules (OLMs) designed for this experiment. Each OLM contains an assessment component (AC) and instructional component (IC). Students are required to make at least one attempt on the AC first, then are allowed to view the IC, and then make subsequent attempts on the AC. Modules 1 and 4 were added for the 2018 implementation. Further, students are randomly assigned into groups that receive two different versions of the IC in module 4, a compare-contrast (CC) task and a tutorial (TT).

measures to estimate whether the observed results are biased by certain extraneous factors, as explained in section III.

\section{METHODS}

\section{A. OLM Sequence Structure}

The study was conducted using online learning modules (OLMs) [5, 15, 16] implemented on the Obojobo platform [17] developed by the Center for Distributed Learning at the University of Central Florida (UCF). Each OLM contains an assessment component (AC) and an instructional component (IC). Students have 5 attempts on the AC which contains 1-2 multiple-choice problems, and must make at least one attempt before being allowed to access the IC. The IC contains instructional text, figures, and/or practice questions which show the solution to the $\mathrm{AC}$ problem on the first attempt either directly (modules 1,3 , and 4 ) or through a series of scaffolding problems (module 2). A student must either pass the $\mathrm{AC}$ or use up all 5 attempts on one module before being allowed access the next module in an OLM sequence. Students' interaction with each OLM can be roughly divided into three stages. In the pre-study (Pre) stage, students must make one or more attempts on the AC prior to accessing the IC. Those who failed during the Pre stage can study the IC during the Study stage, before going into the post-study (Post) stage to make additional attempts on the AC. In $80 \%$ of cases, students accessed the IC of each module only once. In the rest of the cases, their two longest IC access events were treated as a single access event, and the rest of the shorter events were neglected from analysis.

Each student is given a total of 5 attempts on each module, one of which is required to be used before accessing the IC. In this study, a student is counted as passing in the Pre stage if the student correctly answers all problems in the $\mathrm{AC}$ within 2 attempts Alternatively, the student is counted as passing in the Post stage if they did not pass in the Pre stage and then answered correctly within 2 additional attempts after accessing the IC. 
TABLE I. Number of students participating in each OLM sequence, either Rotational Kinematics (RK) or Angular Momentum (AM), in each year, either 2017 or 2018 . Since some students do not complete every module, we report both the number of students who accessed any module and the number who accessed every module.

\begin{tabular}{cccc}
\hline \hline Year & Sequence & $N$ (any module) & $N$ (every module) \\
\hline \multirow{2}{*}{2017} & RK & 210 & 203 \\
& AM & 202 & 200 \\
\hline \multirow{2}{*}{2018} & RK & 206 & 199 \\
& AM & 195 & 191 \\
\hline \hline
\end{tabular}

\section{B. Study Setup}

In Fall 2017, two sequences each containing 3 OLMs (specifically, modules 2, 3, and 5 in Fig. 1) were assigned to roughly 200 students (see Table I) enrolled in a calculusbased introductory physics class at UCF. The ACs of each OLM contain one problem that can be solved using the same physics principle. The IC of the first OLM contains an online tutorial for the problem in the AC, developed by DeVore and Singh $[18,19]$. The IC of the second OLM contains a worked solution to the AC problem, and the IC of the last OLM is empty since the last module is intended to serve the role of a quiz. The first sequence is on rotational kinematics (RK), involving Atwood machine type problems with blocks hanging from massive pulleys. The second sequence is on conservation of angular momentum (AM), involving angular collision problems such as a girl jumping onto a merry-go-round.

The two OLM sequences were modified as follows and implemented again in Fall 2018 by the same instructor to roughly another 200 students (see Table I). To investigate RQ1, we added a new module (module 4 in Fig. 1) in the 2018 implementation between the worked-example module and the quiz module. The AC for this module consists of a new problem that shares the same deep structure as the problem in the previous module, but differs in surface features. The IC for this module comes in two different formats. The compare-contrast (CC) format asks students to first study the solutions of current module's AC (that they just attempted) and of the previous module's AC, then answer 2-3 practice questions comparing the similarities and differences in the solutions of both problems. Each question asks students to select from a list of physics equations the ones that can either be applied to both problems, or are only applicable to one of the problems. In the tutorial (TT) format, the problem in the $\mathrm{AC}$ is broken down into 2-3 tutorial-style sub-problems with solutions given. The student population is divided into two groups with matching average score on a previous midterm exam. Each group is presented with one format of the IC in the RK sequence, and the other format in the AM sequence. RQ1 can be answered by comparing the performance of students in the two groups on the AC of the Quiz module.

To investigate $\mathbf{R Q 2}$, we require students to complete an "on-ramp" module (module 1 in Fig. 1) prior to accessing the tutorial module, with the intention to develop or refresh one or more basic procedural skills necessary for solving the problem in the tutorial module. For the RK sequence, the onramp module presents students with two of the simplest form of Atwood machine problems, involving one or two blocks hanging at the same radius from a single massive pulley. Those problems are intended for students to focus on practicing writing down multiple parallel equations, including applying Newton's second law to both the hanging blocks and the pulley and stating the constraint conditions, without being distracted by a more complicated problem setup. The problems are placed in the AC of the module, while their solutions are in the IC. For the AM sequence, students commonly have difficulty with calculating both the magnitude and the sign of the angular momentum of an object traveling in a straight line about a point that lies off of the line. Therefore, the on-ramp module involves practice problems focusing on developing that particular skill. RQ2 can be answered by measuring students' performance on their AC attempts on the following two modules (tutorial and worked example), especially in the Pre stage of each module, and comparing the performance of the Fall 2018 student population with that of the Fall 2017 population, who did not receive the on-ramp module.

Data analysis, statistical testing and visual analysis are conducted using $\mathrm{R}$ [20] and the tidyverse package [21].

\section{RESULTS}

To answer RQ1, we calculated and compared the passing rates on the Quiz module of Fall 2018 students subjected to either the CC or the TT conditions on module 4, and found no statistically significant differences between the two groups in either sequence. Considering that some students may not have fully engaged with the IC of module 4, we conducted the same comparison among students who spent a "sufficient" amount of time studying the IC of module 4. Since the distribution of study duration on the IC is approximately lognormal, we considered two definitions for "sufficient" time studying the IC: 1) more than the log-mean of study duration and 2) more than one standard deviation below the logmean of study duration. In neither OLM sequence did we find any statistically significant differences between the two conditions in the passing rates of any ACs, including the Post stage of module 4 and the Quiz module.

To investigate the effects of the on-ramp module and answer RQ2, we first plotted passing rates on the Pre and Post stage AC attempts of each module in Fig. 2 for students in both Fall 2017 and Fall 2018. The Pre passing rates are calculated based on all students who attempted the module, while the Post passing rates are calculated only for those who failed in Pre (i.e., the pass rates reported for Post are not cumulative). Note that since modules 1 and 4 were added in 2018, the 2017 population does not have data for those modules. Furthermore, since the IC of the Quiz module was empty, we did not distinguish between Pre and Post stage attempts when analyzing its passing rates. Lastly, since we did not find any differences between the CC and TT conditions on module 4, the two groups were combined in this analysis. 

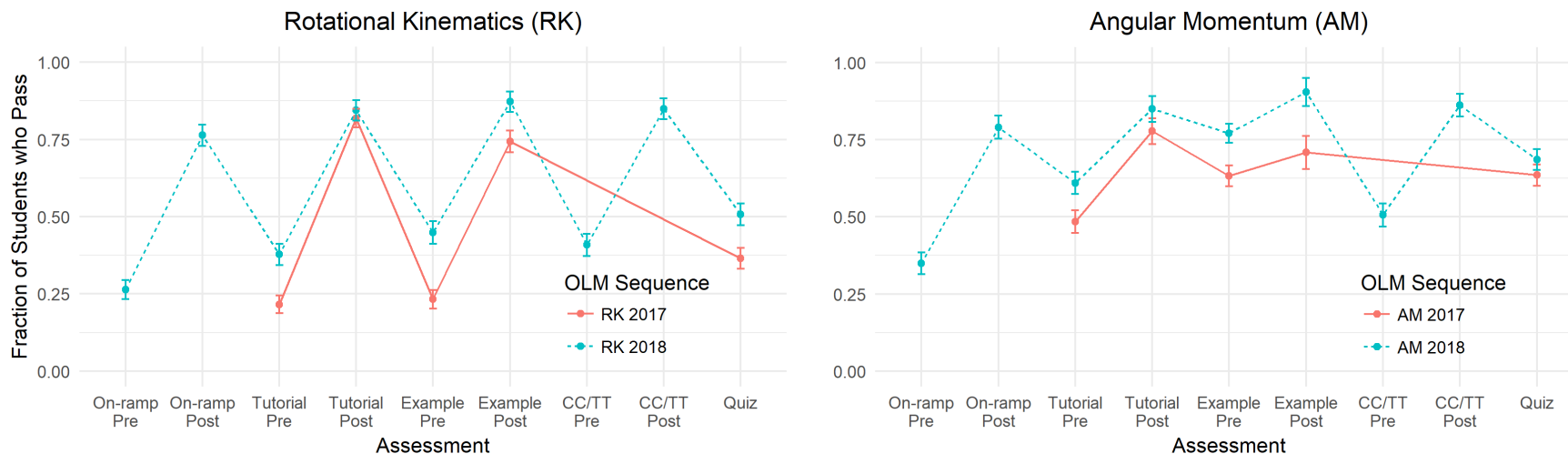

FIG. 2. The passing rates on each assessment for the two OLM sequences. Passing rates are calculated as the fraction of students who attempt the assessment and pass within two attempts, and plotted along with their standard error. Passing rates on Post assessments are calculated only for those who did not pass in Pre.

TABLE II. Findings of student performance differences from 2017 to 2018 . The left side compares overall pass rates from 2017 to 2018 while the right compares pass rates separately for students whose first attempt duration was $\leq 30 \mathrm{~s}$ or $>30 \mathrm{~s}$. Due to the elevated Type II error caused by the large number of tests performed, we report the $p$-values adjusted by the Holm-Bonferroni method [22]. Tests with $p_{\text {adj }}>0.10$ are not shown except AM Tutorial Pre and AM Tutorial Post, which are included as context for the corresponding entries on the right side.

\begin{tabular}{|c|c|c|c|c|c|c|c|c|c|}
\hline Population & Assessment & $\begin{array}{c}2017 \\
\text { Pass Rate }\end{array}$ & $\begin{array}{c}2018 \\
\text { Pass Rate }\end{array}$ & $p_{\text {adj }}$ & $\begin{array}{l}\text { 1st Attempt } \\
\text { Duration }\end{array}$ & Assessment & $\begin{array}{c}2017 \\
\text { Pass Rate }\end{array}$ & $\begin{array}{c}2018 \\
\text { Pass Rate }\end{array}$ & $p_{\text {adj }}$ \\
\hline All & RK Tutorial Pre & $22 \%$ & $38 \%$ & 0.01 & $>30 \mathrm{~s}$ & RK Tutorial Pre & $20 \%$ & $40 \%$ & 0.02 \\
\hline All & RK Example Pre & $23 \%$ & $45 \%$ & $<0.01$ & $>30 \mathrm{~s}$ & RK Example Pre & $22 \%$ & $51 \%$ & $<0.01$ \\
\hline All & AM Tutorial Pre & $49 \%$ & $61 \%$ & 0.48 & $\leq 30 \mathrm{~s}$ & AM Tutorial Pre & $42 \%$ & $68 \%$ & 0.06 \\
\hline All & AM Example Pre & $63 \%$ & $77 \%$ & 0.12 & $\leq 30 \mathrm{~s}$ & AM Example Pre & $48 \%$ & $75 \%$ & 0.05 \\
\hline
\end{tabular}

As shown in Fig. 2, Fall 2018 students had higher passing rates than Fall 2017 students in every module common to both years, though many of these differences were not statistically significant. To examine the significance of the differences, we conducted Fisher's exact test on each pair of passing rates common to both 2017 and 2018. Due to the large number of tests performed throughout the analysis which can cause elevated Type II error, the $p$-values were adjusted using the Holm-Bonferroni method [22]. Of the ten such tests conducted, only two were significant after $p$-adjustment, namely RK Tutorial Pre and RK Tutorial Post, as seen on the left side of Table II. AM Tutorial Pre and AM Tutorial Post, despite being non-significant after $p$-adjustment, are also included as context for the corresponding entries on the right side of the table. To ensure that the observed differences were not caused by the student population in 2018 being in general stronger than the student population in 2017, we checked their scores on four common problems given on a classroom exam that was administered shortly before students were assigned the modules. On none of the problems did 2018 students outperform the 2017 students.

As mentioned in section I, one extraneous factor that could have contributed to the observed differences is that some students may have obtained the answers to the problems from other sources. A thorough and complete investigation of such phenomena, such as in [13] is far beyond the scope of the current paper. However, we can still make a less precise estimation by assuming that students who spent less than 30 seconds are much less likely to have actually solved the problem. If the observed improvement in performance were due to increase in problem solving skills, then the difference should not be observed among students who spent less than $30 \mathrm{sec}-$ onds on their attempts.

In order to determine whether or not the improvements observed in Fig. 2 occurred among students who seriously attempted the problem, we performed the same analysis on students who spent $\leq 30 \mathrm{~s}$ and $>30 \mathrm{~s}$ on their $\mathrm{AC}$ attempts separately, and listed the results that are either significant or marginal at the $\alpha=0.05$ level after $p$-value adjustment on the right side of Table II. On both RK Tutorial and RK Example modules, the differences in Pre stage passing rates remain significant for the $>30$ s population, but not the $\leq 30$ s population. On the other hand, for the same two modules in the AM sequence, the differences were only marginally significant for the $\leq 30$ s population after $p$-value adjustment.

\section{DISCUSSION}

For RQ1, we found no statistically significant differences in subsequent Quiz module performance between students subjected to the CC and TT conditions. There are several possible explanations for this observation. First, unlike many previous studies that relied on explicit self-explanation [6, 9, 10], the current implementation of the compare-contrast tasks via multiple choice problems may not be sufficient to engage many students in an authentic and productive comparecontrast process. Second, the current compare-contrast prob- 
lems focused on identifying the correct mathematical expression applicable in each situation which may have encouraged rote memorization and a "plug-and-chug" approach. Future implementations may explore more effective ways to focus on contrasting surface feature differences and comparing deep structure similarities. Finally, the current study design used the Quiz module as the sole assessment for detecting potential differences between the $\mathrm{CC}$ and TT conditions. The problems in the Quiz modules could have insufficient discrimination power to detect any differences between the groups.

For RQ2, the consistent and significant increase in passing rate from 2017 to 2018 in the Pre stages of the RK Tutorial and RK Example modules serve as evidence for the benefit of the on-ramp module, since students have not yet accessed the ICs of the modules while in the Pre stage. The fact that the improvement is significant for the $>30$ s group and not for the $\leq 30$ s group further suggests that the improvement is likely due to actual increase in problem solving ability, rather than extraneous factors. On the other hand, the overall differences in the AM sequence are not only non-significant after $p$-adjustment, but also mainly due to the increased passing rate in the $\leq 30 \mathrm{~s}$ group as seen in Table II. This observation suggests that the benefit of an on-ramp module is not uniform, and can depend on various other factors such as content, assessment and the implementation. However, we note that in both the RK and AM sequences, Fig. 2 shows several 10-15\% passing rate improvements in 2018 over 2017. The differences are not all significant after p-adjustment in the current study, thought some of them may turn out to be meaningful improvements in future studies with larger sample size.

Another noteworthy observation is that, despite the addition of two new modules, the improvement in passing rates over the 2017 population on the RK Quiz module is marginal at best (and non-significant after $p$-adjustment), and insignificant for the AM Quiz module. For the RK sequence, it is likely that Atwood machine problems remain very challenging for the student population involved, since the RK Quiz module passing rate remains at $50 \%$, despite the improvements seen on previous modules. For the AM sequence, however, it could be that the problems are not challenging enough, since the AM Quiz module passing rate in 2017 was already at $75 \%$, leaving little room for further improvement. In addition, there is a noticeable drop in passing rate on AM Module 4 Pre, compared to the Pre stage of the two preceding modules. More careful analysis shows that most wrong answers concentrated on one of the distractors which is written in a potentially confusing way. This may have negatively impacted the effectiveness of AM Module 4.

Overall, our observations suggest that even when instructional resources are created based on well established principles from learning science, their effectiveness can be highly sensitive to implementation details and other factors such as the difficulty of the content. This observation calls for new methods that can quickly evaluate the effectiveness of new instructional materials and pedagogical innovations in order to reliably improve the quality of instruction. The current study demonstrated two such methods using OLM sequences and analysis of student log data. The first is a controlled AB experiment conducted in a single course, similar to the design in two previous studies $[5,23]$. The second method involves implementing improved instructional design in the same class for consecutive years.

Compared to conventional clinical or classroom experiments, both new methods are much easier to implement and replicate, allow more flexibility in study design, and provide rich information on students' learning behavior that could support in-depth data analysis. The first method has the advantage of reducing the number of extraneous impacting factors, but it is also more disruptive for students and more logistically complicated to implement. It also resulted in a smaller sample size since each group contains only half of the class population. The second method provides a sample size of two class populations and is far less intrusive to implement as part of normal instruction. However, the results are subjected to the influence of more extraneous factors, which require sophisticated data analysis procedures to validate.

\section{A. Future directions}

Several follow up analyses can be carried out in the future to gain new insight into the current results and address some of the shortcomings of the current study. For instance, the current analysis for most part did not take into account the variation in students' level of engagement with the instructional material, as some students spent significantly less time studying the IC than others. Future analysis could probe the relationship between the level of engagement with the IC and students' performance on subsequent problem-solving task.

Furthermore, since the passing rate on the Pre stages for each module are used as a measure of the effectiveness of instruction, future studies should investigate what fraction of students took the initial attempts seriously before accessing the IC. Preliminary analysis on the duration of the attempts has shown that some students seem to consistently submit a random guess on their first attempt, perhaps since they know that the IC will guide them towards solving the AC problem. Since this type of behavior significantly reduces the validity of using Pre stage attempts as assessments, future studies need to explore methods to encourage serious problem solving, such as providing small amounts of credit incentives.

Finally, an important topic for future analysis is to examine whether the observed benefits of new interventions are uniform across the student population or selectively benefit students of, e.g., certain demographic backgrounds.

\section{ACKNOWLEDGMENTS}

The authors would like to thank the Learning Systems and Technology team at UCF for developing the Obojobo platform. This research is partly supported by NSF Award No. DUE 1845436. 
[1] H. S. Broudy, Types of knowledge and purposes of education, in Schooling and the Acquisition of Knowledge, edited by R. C. Anderson, R. J. Spiro, and W. E. Montague (Routledge, 1977) pp. $1-17$.

[2] D. K. Detterman, The case for the prosecution: Transfer as an epiphenomenon, in Transfer on Trial: Intelligence, Cognition, and Instruction, edited by D. K. Detterman and R. J. Sternberg (Ablex Publishing, 1993) pp. 1-24.

[3] J. D. Bransford and D. L. Schwartz, Rethinking transfer: A simple proposal with multiple implications, Review of Research in Education 24, 61 (1999).

[4] M. T. H. Chi, R. Glaser, and E. Rees, Expertise in problem solving: Advances in the psychology of human intelligence, in Advances in the Psychology of Human Intelligence, Vol. 1, edited by R. J. Sternberg (1982) pp. 1-75.

[5] Z. Chen, K. M. Whitcomb, and C. Singh, Measuring the effectiveness of online problem-solving tutorials by multi-level knowledge transfer, in Physics Education Research Conference 2018, PER Conference, edited by A. Traxler, Y. Cao, and S. Wolf (Physics Education Research Topical Group and the American Association of Physics Teachers, Washington, DC, 2018).

[6] D. Gentner, J. Loewenstein, and L. Thompson, Learning and transfer: A general role for analogical encoding, Journal of Educational Psychology 95, 393 (2003).

[7] D. L. Schwartz, C. C. Chase, M. A. Oppezzo, and D. B. Chin, Practicing versus inventing with contrasting cases: The effects of telling first on learning and transfer, Journal of Educational Psychology 103, 759 (2011).

[8] J. Roelle and K. Berthold, Effects of comparing contrasting cases and inventing on learning from subsequent instructional explanations, Instructional Science 44, 147 (2016).

[9] R. Badeau, D. R. White, B. Ibrahim, L. Ding, and A. F. Heckler, What works with worked examples: Extending selfexplanation and analogical comparison to synthesis problems, Physical Review Physics Education Research 13, 1 (2017).

[10] E. Kuo and C. E. Wieman, Toward instructional design principles: Inducing Faraday's law with contrasting cases, Physical Review Physics Education Research 12, 010128 (2016).

[11] B. D. Mikula and A. F. Heckler, Framework and implementation for improving physics essential skills via computer-based practice: Vector math, Physical Review Physics Education Research 13, 010122 (2017).

[12] N. T. Young and A. F. Heckler, Observed hierarchy of student proficiency with period, frequency, and angular frequency, Physical Review Physics Education Research 14, 10104 (2018).

[13] G. Alexandron, J. A. Ruiperez-Valiente, Z. Chen, Pedro J. Muñoz-Merino, and D. E. Pritchard, Copying @ scale: Using harvesting accounts for collecting correct answers in a MOOC, Computers \& Education 108, 96 (2017).

[14] Chegg, Chegg Inc., Santa Clara, CA (2019).

[15] Z. Chen, G. Garrido, Z. Berry, I. Turgeon, and F. Yonekura, Designing online learning modules to conduct pre- and posttesting at high frequency, in Physics Education Research Conference 2017, PER Conference (Physics Education Research Topical Group and the American Association of Physics Teachers, Cincinnati, OH, 2017) pp. 84-87.

[16] Z. Chen, S. Lee, and G. Garrido, Re-designing the structure of online courses to empower educational data mining, Proceedings of the 11th International Conference on Educational Data Mining, EDM 2018, Buffalo, NY, USA, July 15-18, 2018, (2018).

[17] Z. Berry, I. Turgeon, and F. Yonekura, Obojobo Next (2019).

[18] S. DeVore, E. Marshman, and C. Singh, Challenge of engaging all students via self-paced interactive electronic learning tutorials for introductory physics, Physical Review Physics Education Research 13, 010127 (2017).

[19] C. Singh and D. Haileselassie, Developing problem-solving skills of students taking introductory physics via web-based tutorials, Journal of College Science Teaching 39, 42 (2010).

[20] R Core Team, R: A Language and Environment for Statistical Computing, R Foundation for Statistical Computing, Vienna, Austria (2019).

[21] H. Wickham, tidyverse: Easily Install and Load the 'Tidyverse' (2017), R package version 1.2.1.

[22] Y. Benjamini and Y. Hochberg, Controlling the false discovery rate: a practical and powerful approach to multiple testing, Journal of the Royal Statistical Society: Series B (Methodological) 57, 289 (1995).

[23] Z. Chen, N. Demirci, Y.-J. Choi, and D. E. Pritchard, To draw or not to draw? Examining the necessity of problem diagrams using massive open online course experiments, Physical Review Physics Education Research 13, 010110 (2017). 